\title{
Genetic diversity and main functional composition of Lingzhi strains from main producing areas in China
}

\author{
Yuan-chao Liu ${ }^{1,2 \dagger}$, Xiao-cui Tang ${ }^{2,3+}$, Hui-ping Hu${ }^{2 \dagger}$, Di-ling Chen², Yi-zhen Xie ${ }^{2,3}$, Xiao-wei Liang ${ }^{2}$, Xiang-min Li ${ }^{2}$, \\ Chun Xiao ${ }^{2}$, Long-hua Huang ${ }^{2}$ and Qing-ping Wu $\mathbf{u}^{1,2^{*}}$ (D)
}

\begin{abstract}
In this study, we used genotyping by sequencing (GBS) to examine the genetic diversity of 22 strains of Lingzhi and the quality differences in 15 fruit bodies of Lingzhi from different Chinese regions. The phylogenetic trees of 22 strains were constructed based on ITS (Internal transcribed spacer) and SNP (single nucleotide polymorphism). Moisture, ash, water-soluble extracts, alcohol-soluble extracts, polysaccharides, and triterpenoids from 15 fruit bodies of Lingzhi were detected and analyzed based on Chinese Pharmacopoeia and the US Pharmacopoeia references. Moreover, the monosaccharide composition of polysaccharides was studied using PMP-HPLC, and the effect of polysaccharides on the proliferation rate of splenocytes was investigated in vitro. The identification results of these strains by the phylogenetic trees which were constructed based on ITS sequences and SNPs showed that most of the strains applied in the main producing areas of Lingzhi in China were accurate except for a few inaccurate strains. The moisture, ash, water and alcohol soluble extractive, polysaccharide and triterpenoid content of all samples were meet the requirements of the Chinese Pharmacopoeia, while the polysaccharide and triterpenoid content of less than half of the samples meet the requirements of the U.S. Pharmacopoeia. The polysaccharide extracted from these samples have different effects on the proliferation rate of spleen cells. To sum up, this is the first study that reported on the differences in Lingzhi strains from the main producing areas in China. The quality of some fruit bodies did not meet the pharmacopeia requirements, and wrong strains were used in some production areas; thus, strains should be given special attention before legal processing.
\end{abstract}

Keywords: Lingzhi, Ganoderma lucidum, Polysaccharides and triterpenoids, Monosaccharide, GBS technique, SNP, Phylogenetic tree

\section{Introduction}

Ganoderma lucidum (also known as Lingzhi or Ganoderma lingzhi in China) is an edible and medicinal fungus that belongs to the genus Ganoderma, family Polyporaceae in Basidiomycete (Dai et al. 2013; Zhou et al. 2015). As a traditional Chinese herb, it has been applied

\footnotetext{
*Correspondence: wuqp203@163.com

${ }^{\dagger}$ Yuan-chao Liu, Xiao-cui Tang and Hui-ping Hu contributed equally to this paper

${ }^{1}$ School of Biology and Biological Engineering, South China University of Technology, Guangzhou, China

Full list of author information is available at the end of the article
}

for 2300 years to treat various human conditions. It can enrich the yin and nourish the kidney, relieve cough and asthma, prolong life, and support healthy energy (Paterson 2006; Li et al. 2012, 2016). The most abundant biologically active substances in Lingzhi are polysaccharides, nucleosides, triterpenoids, peptides, sterols, protein, and alkaloids (Sun et al. 2014). Polysaccharides and triterpenoids are considered to be the main medicinal components. Studies have shown that Lingzhi protects the liver; it has anti-tumor, anti-inflammatory, anti-virus, antioxidation, anti-aging, anti-radiation effect; it regulates the endocrine system, enhances immunity. Moreover, it
Springer Open
C The Author(s) 2021. Open Access This article is licensed under a Creative Commons Attribution 4.0 International License, which permits use, sharing, adaptation, distribution and reproduction in any medium or format, as long as you give appropriate credit to the original author(s) and the source, provide a link to the Creative Commons licence, and indicate if changes were made. The images or other third party material in this article are included in the article's Creative Commons licence, unless indicated otherwise in a credit line to the material. If material is not included in the article's Creative Commons licence and your intended use is not permitted by statutory regulation or exceeds the permitted use, you will need to obtain permission directly from the copyright holder. To view a copy of this licence, visit http://creativecommons.org/licenses/by/4.0/. 
can reduce blood glucose, reduce uric acid, blood lipids, and regulate gut microbiota (Tang et al. 2005b; Yan et al. 2010; Wasser 2014; Wang et al. 2015; Chang et al. 2017; Yan et al. 2017)

Ganoderma spp is globally distributed, being predominant in the tropical and subtropical regions, including China (Dai et al. 2013; Richter et al. 2015). Besides, Lingzhi has also been artificially cultivated in Zhejiang Longquan, Heilongjiang, Jilin, Hebei, Shandong, Anhui Huoshan, Jiangsu, Jiangxi, Hunan, Guizhou, Fujian, and Guangxi provinces (Zhao 1989; Zhang 2013), thus making China the global leading exporter of Lingzhi (Jin et al. 2016). According to the available statistics, the yield of Lingzhi and its spore powder was about 12 million tons in 2015, and the output value was 1.6 billion dollars, accounting for about $75 \%$ and $30 \%$ of global values. However, the quality of Lingzhi may vary based on differences in strain, production region, growing condition, cultivation techniques, and harvesting time ( $\mathrm{Lu}$ et al. 2012; Chen et al. 2016; Zhao 2020). Thus, accurate identification and quality assessment of G. lingzhi are very important. Considering the uncertainty of the morphological characteristics of fruit body in different growth periods, molecular biological methods are commonly applied for quality assessment of G. lingzhi (Wang et al. 2011). According to related reports, ITS 2 (internal transcribed spacer 2) (Liao et al. 2015), ITS (Su et al. 2007), RAPD (random amplified polymorphic-DNA) (Wang et al. 2011), and SCAR sequence charactered amplified region) techniques (Xu et al. 2008) were used to identify Ganoderma (Lingzhi) fruit bodies of different origins. The results showed that these methods have certain effectiveness but could not distinguish between similar samples. Thus, a more accurate method should be developed.

Over the years, several high-throughput sequencing methods combined with bioinformatics analysis have been developed, which can free up tedious PCR workload and improve detection efficiency and accuracy. Highthroughput sequencing techniques based on restriction enzyme digestion include GBS (Genotyping By Sequencing), RRLs (reduced-representation libraries), CRoPS (complexity reduction of polymorphic sequences), and RAD-Seq (restriction-site-associated DNA sequencing). GBS is a cost-effective approach widely applied in SNP (single nucleotide polymorphism) detection and genotyping research (Sonah et al. 2013). These techniques can be applied in molecular marker development and genotyping to model species with high-quality reference genomic sequences and the non-reference genomic species (Xiao et al. 2014).

In this study, we used the GBS technique to examine the genetic relationship of 22 strains of Lingzhi and ITS sequences. Moisture, ash, water-soluble extracts, alcohol-soluble extracts, polysaccharides, and triterpenoids of 15 fruit bodies of Lingzhi were detected and analyzed based on Chinese Pharmacopoeia and the US Pharmacopoeia references. Moreover, the monosaccharide composition of polysaccharides was studied using PMP-HPLC, and the effects of polysaccharides on the proliferation rate of splenocytes were investigated in vitro.

\section{Materials and methods \\ DNA extraction of mycelium and ITS-PCR}

The strains and the fruit bodies (part of) cultivated by the strain from the main producing areas of G. ling$z h i$ in China were investigated. Fifteen fruit bodies and 22 strains of Lingzhi were collected (Additional file 1: Table S1). Strains were inoculated into the sterilized liquid fermentation medium (PD, autoclaved at $121{ }^{\circ} \mathrm{C}$ and $98 \mathrm{kPa}$ for $20 \mathrm{~min}$ ) in the shaker at $27^{\circ} \mathrm{C}, 150 \mathrm{rpm}$ for 9 days. Samples were then centrifuged at $8000 \mathrm{rpm}$ to discard the medium, and mycelia were washed with pure water three times and prepared for use.

The TaKaRa MiniBEST Plant Genomic DNA Extraction Kit and PCR mix [Prime STAR Max Premix $(2 \times)$ bought from Takara Biomedical Technology (Beijing) Co., Ltd.] were used for extracting total genomic DNA and PCR of 22 strains, respectively. The ITS primers and PCR protocol were applied as previously described (White et al. 1990). The primers synthesized and PCR products sequencing were obtained from Beijing Genomics Institute (BGI).

\section{Construction of phylogenetic tree based on ITS sequences}

Parts of Sequences in this study used for phylogenetic analysis were downloaded from GenBank. Sequences were aligned using Clustal X (Thompson et al. 1997) and edited by Bioedit (Hall 1999). Phylogenetic analyses were performed with MEGA v7.0.26 (Kumar et al. 2016). A phylogenetic tree was constructed using the maximum likelihood method. Bootstrap values were calculated from 1000 replicates. Branches corresponding to partitions reproduced in less than $50 \%$ bootstrap replicates were collapsed. All positions containing gaps and missing data were used from the dataset.

\section{GBS sequencing and genetic structure analysis}

Guangzhou Jidi'ao Biotechnology Co., Ltd preferred a GBS library construction and sequencing of 22 Lingzhi mycelia samples. The genome of each sample was digested with restriction endonuclease (GAATTC, CATG), and then T4 DNA ligase was used to ligate the adapter with a barcode. A small fragment library (250$550 \mathrm{bp}$ ) was constructed for double-ends sequencing by PE125. High-quality reads which were obtained by strict 
quality control, including removing the reads with $\mathrm{N}$ ratio of more than $10 \%$ and the low-quality reads, were mapped to the reference genomes with mem algorithm using BWA v0.7.12(Li and Durbin 2009); Ganoderma lucidum G.260125-1 was used as a reference genome (Chen et al. 2012), mapping parameter was $-\mathrm{k} 32-\mathrm{M}$. The results were marked using Picard v1.119 (Wysoker et al. 2014). Unified Genotyper module of the software GATK v3.4 (Mckenna et al. 2010) was used to handle the mapping file for Variant detection of multiple samples. The detected variants were filtered by Variant Filtration with filter parameters -Window4, -filter "QD $<4.0 \| \mathrm{FS}>60.0$ II MQ<40.0", - G_filter" GQ<20". ANNOVAR (Wang et al. 2010) was used to annotate the detected Variants. Plink (Purcell et al. 2007) and Frappe (Tang et al. 2005a) were used to infer the population structure within the different Lingzhi with different $\mathrm{K}$ values (from 2 to 10). Principal component analysis (PCA) was accomplished with R language (http://www.r-project.org/) based on the SNP between individuals. The phylogenetic tree was constructed by TreeBeST v1.9.2 (Vilella et al. 2009) with a neighbor-joining method based on the SNP. The samples were clustered to analyze the genetic relationship among the populations.

\section{Component detection and analysis}

Moisture, ash, polysaccharides, and triterpenoids of samples from Lingzhi fruit bodies were detected using the latest version of Chinese Pharmacopoeia (Committee Chinese Pharmacopoeia 2015) and US (Convention the United States Pharmacopeial 2016); water and alcohol soluble extract was slightly modified based on the Chinese Pharmacopoeia. The colorimetric method by an ultraviolet spectrophotometry was used to determine the content of polysaccharides and total triterpenes at $490 \mathrm{~nm}$ and $546 \mathrm{~nm}$, respectively. The phenol-Sulfuric Acid method was selected for the detection of polysaccharides, and Oleanolic acid was used as a reference substance for the detection of total triterpenes. Data analysis and graphs constructed were performed using Excel (Microsoft 365).

\section{Effects of polysaccharides on proliferation ability of splenocytes in vitro}

Balb/c male nude mice, 8 weeks old, weighing $20-25$ g, were obtained from Guangdong Medical Laboratory Animal Center, Guangzhou, China. All the animals were housed in the SPF Animal Laboratory of Guangdong Institute of Microbiology, kept an environment with a temperature of $25 \pm 1{ }^{\circ} \mathrm{C}$, relative humidity of $55 \pm 1 \%$, and a light/dark cycle of $12 / 12 \mathrm{~h}$. All experimental protocols were approved by the Animal Ethics Committee of Guangdong Institute of Microbiology, and all experimental procedures were conformed by the National Institutes of Health Guide for the Care and Use of Laboratory Animals. Spleens were removed from mice and immersed in PBS buffer, cut into pieces with scissors, gently ground with a syringe piston. The cells suspension was obtained using a 200-mesh cell filter. The cells filtrate was then centrifuged for $5 \mathrm{~min}$ at RCF $250 \times g$, after which the supernatant was discarded. The rest was mixed with $1 \mathrm{ml} \mathrm{ACK}$ lysate (room temperature) to resuspended cells at room temperature for $5 \mathrm{~min}$. Cells were then mixed with $7 \mathrm{ml}$ precooling PBS, centrifuged for $5 \mathrm{~min}$ at $250 \times g$, and washed twice with cooling PBS.

The effects of polysaccharides on the proliferation ability of splenocytes in vitro were examined using a previously described approach ( $\mathrm{Li}$ et al. 2019) with a slight modification. Spleen cells $\left(6 \times 10^{6}\right.$ cells $\left./ \mathrm{mL}, 100 \mu \mathrm{L}\right)$ were seeded onto 96 -well tissue culture plates. After reaching an $80 \%$ confluence, cells were incubated in polysaccharide $(10 \mu \mathrm{g})$ dissolved in $100 \mu \mathrm{l}$ DMEM $(10 \% \mathrm{FBS}, 100 \mathrm{U} /$ $\mathrm{ml}$ penicillin/streptomycin) and at $37{ }^{\circ} \mathrm{C}$ and $5 \% \mathrm{CO}_{2}$ for $72 \mathrm{~h}$. MTS method was used to measure the OD value at $490 \mathrm{~nm}$ by a microplate reader (MUITISKAN GO, Thermo Scientific). All data obtained were analyzed by Kruskal-Wallis test performed with $\mathrm{R}$ software, version 3.5.3 (R Foundation, Vienna, Austria) (Kaur et al. 2020), p-values of less than 0.05 were considered to indicate statistical significance.

\section{Results}

\section{Sequencing data and phylogenetic tree}

The ITS-rDNA sequences in this study have been uploaded to NCBI, and the GenBank Accession Numbers were MN911326-MN911347. Other sequences, including the outgroup Amauroderma rude, were download from NCBI. Twenty-two strains were sampled for GBS. A total of $3.649 \mathrm{~Tb}$ of clean data and $3.567 \mathrm{~Tb}$ of $\mathrm{HQ}$ clean data after filtration were generated. The effective tags coverage in the genome was $0.19 \%$ (Additional file 1 : Tables S2-S4). The raw data of the GBS sequence has been uploaded to NCBI, and the SRA Accession Number is PRJNA600664.

The phylogenetic tree constructed based on ITSrDNA sequences revealed that 22 Lingzhi strains could be divided into four categories. Among these strains, 16SHD01-02 (Group I) from Liaocheng city, Shandong Province, were clustered in the same category with Ganoderma sessile (and identified as Ganoderma sessile), which is consistent with the reference genome comparison (Additional file 1: Table S5). 16JL01-01 from Jiaohe city, Jilin Province, and 16SHD01-01 from Liaocheng city, Shandong Province, were individually clustered in one small category (Group II). The remaining 19 strains were clustered together with Ganoderma lingzhi, and were 
identified as Ganoderma lingzhi. Among these strains, 16ZHJ03-02, 16SHX02-02, 16ANH02-00, 16ZHJ01-01, $16 \mathrm{ZHJ} 03-00$, and 16ANH01-00 were closely related and clustered in one branch (Group IV); all showed high spore powder production ability. 16SHX02-00, 16ZHJ0301, 16ZHJ01-00, 16ZHJ02-00, 16FJ01-00, and 16SHX0100 were closely related and clustered in another branch (Group III) (Fig. 1).
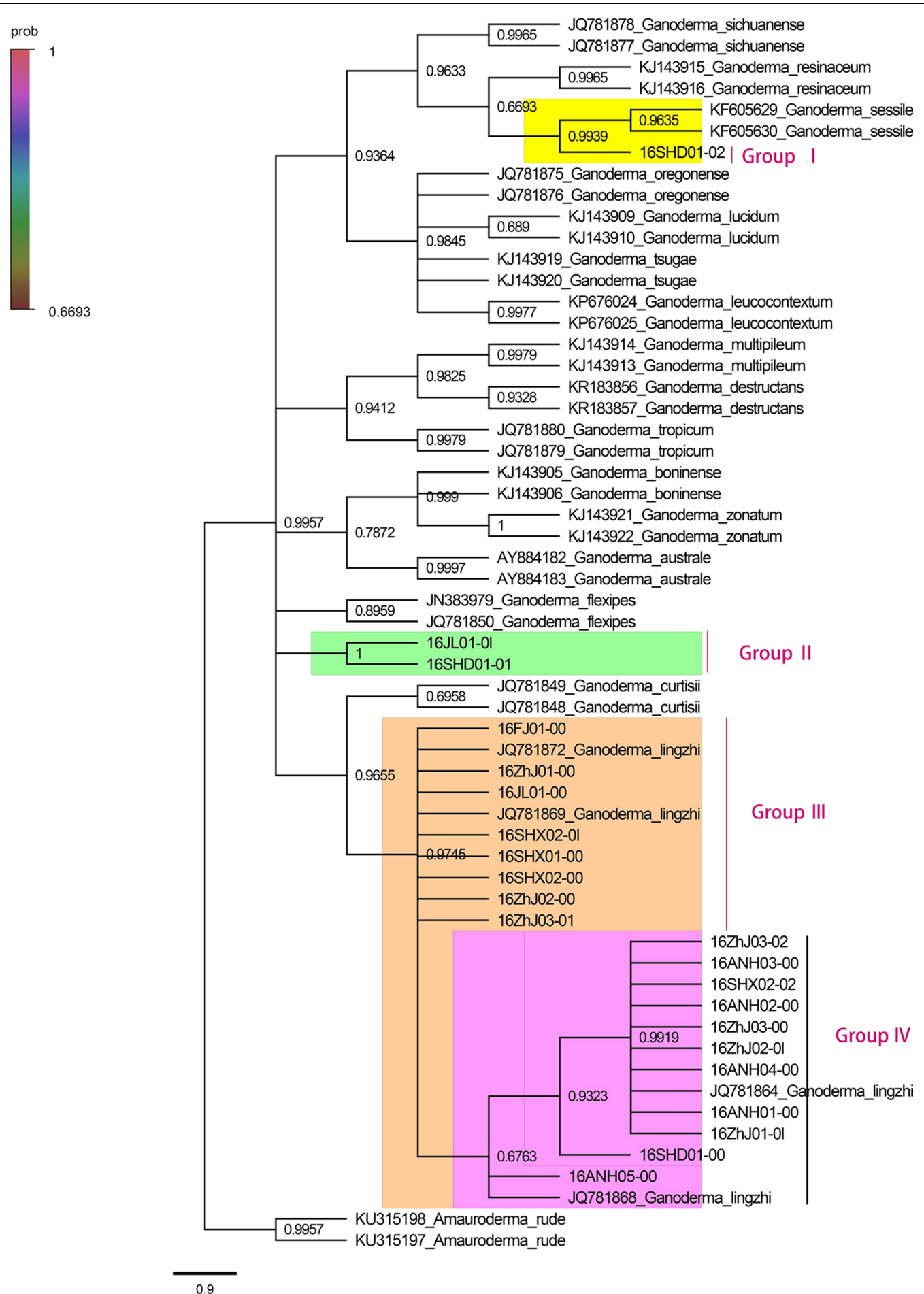

Fig. 1 Phylogenetic tree of 22 lingzhi strains based on ITS sequences 
The principal component analysis (PCA) demonstrated that the first component could differentiate the Group I from Group II, Group III and Group IV, the Group II, Group III, and Group IV was in the second component, but they could be separated significantly. The first and second components explained $58.1 \%$ and $16.4 \%$ of the SNP variances, respectively (Fig. 2). Altogether, these results indicated that Group II, Group III, and Group IV were similar but were significantly different from Group I; this result was consistent with the phylogenetic tree based on ITS sequences, revealing that 16SHD01-02 was not G. lingzhi.

Next, the population structure among the samples with different values for $\mathrm{K}$ (from 2 to 10) was analyzed (Fig. 3). The $\mathrm{K}$ value was determined based on the cross-validation error rate, and the $\mathrm{K}$ value with the lowest cross-validation error rate was the optimal K value (Fig. 4). When $\mathrm{K}$ was 2, the cross-validation error rate was the lowest, which indicated that the best classification strategy for the population was divided into two subgroups.

As 16SHD01-02 was not considered Lingzhi, the strain was removed from the group in the following phylogenetic tree analysis. Based on the effective
SNPs (Additional file 1: Table S6 and Additional file 2: Table S7), the Neighbor-Joining (NJ) phylogenetic trees of the Lingzhi strains across China were constructed. All Lingzhi strains formed three different clades (Fig. 5; the two main branches strains almost contained all Lingzhi strains, 16ANH01-00, 16ANH02-00, 16ANH03-00, 16ANH04-00, 16ANH05-00, 16SHX02-02, 16ZHJ01-01, 16ZHJ03-00, 16ZHJ02-01, 16SHD01-00. 16ZHJ03-02 formed one of the main branches, which was similar to the group IV, while 16FJ-01-00, 16JL01-00, 16SHD0101, 16SHX01-00, 16SHX02-00, 16SHX02-01, 16ZHJ01$00,16 \mathrm{ZHJ} 03-01$ and $16 \mathrm{ZHJ} 02-00$ formed another main branch, which was similar to the group III. However, 16)L-01-01 formed a single branch alone.

\section{The result of the component determination Moisture and ash content}

According to the 2015 edition of the Chinese Pharmacopoeia and the US, moisture content of G. lucidum should not exceed $17 \%$. In this study, the moisture contents of fruit bodies from 15 samples of Lingzhi were low. The lowest moisture content was observed in 16SHX02-00 (1.47\%), and the highest content in 16ZHJ03-00 (7.28\%).

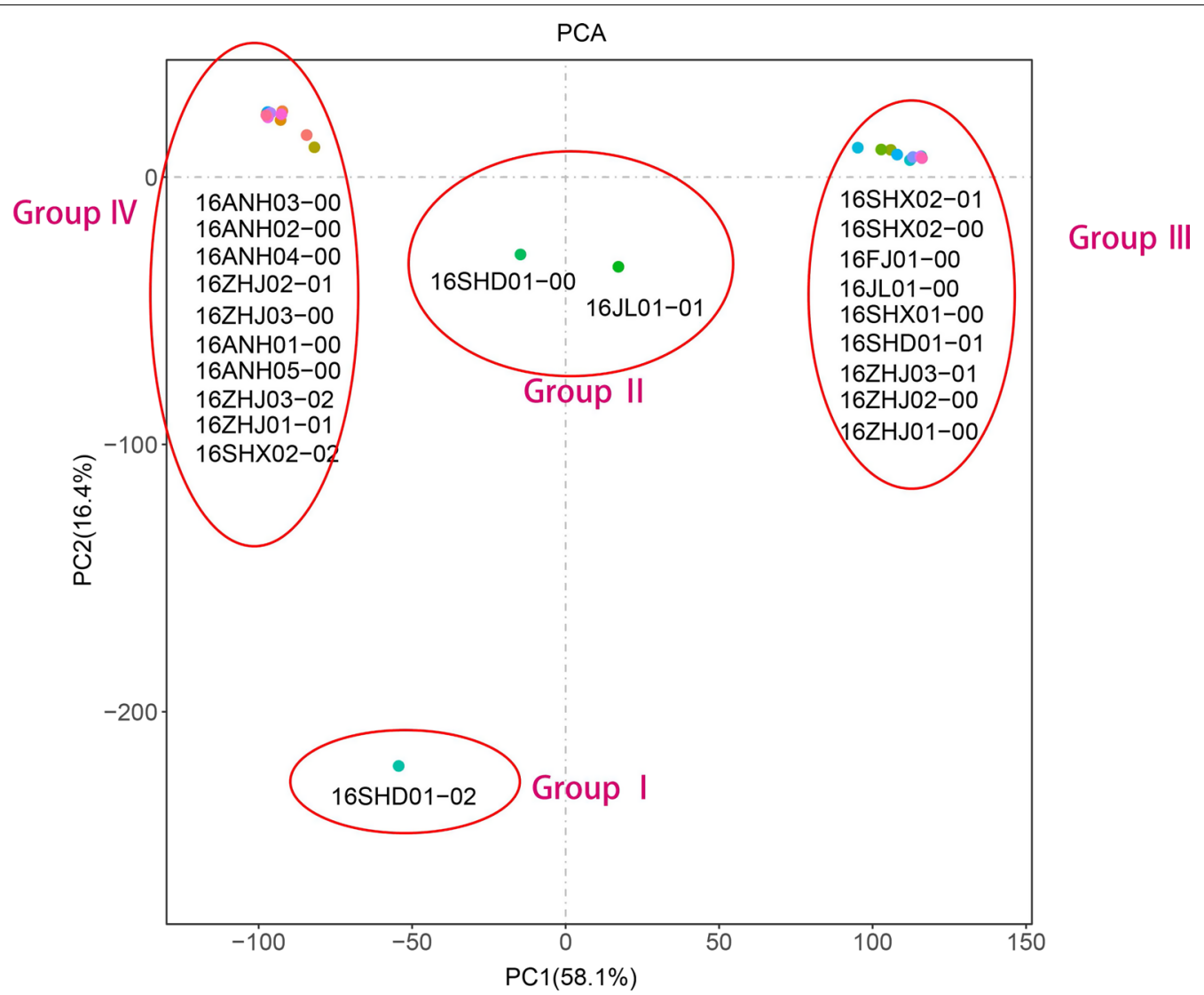

Fig. 2 Principal component analysis of 22 lingzhi strains based on SNP 


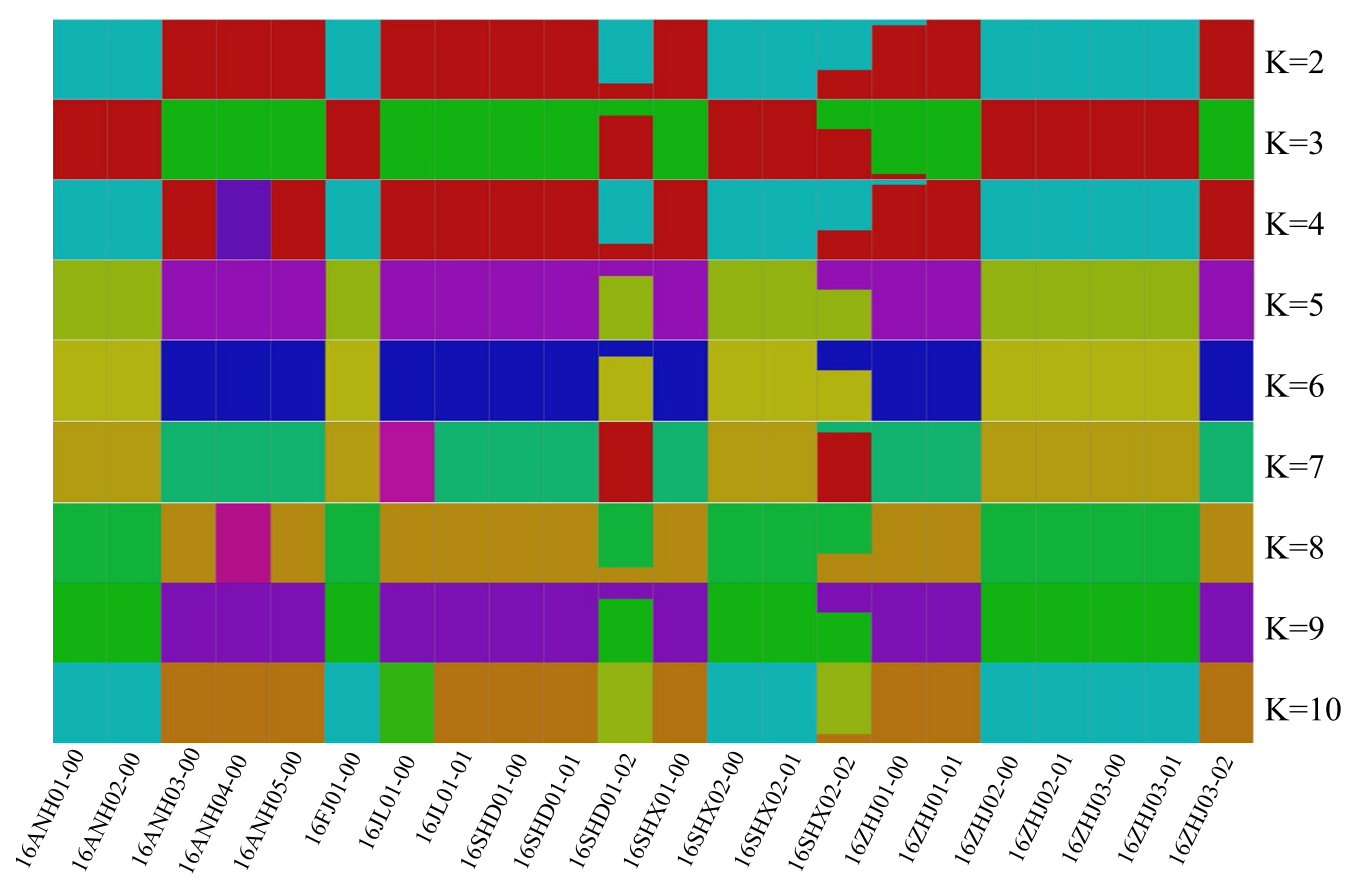

Fig. 3 Population structure analysis of 22 lingzhi strains based on SNP

In addition, according to the Chinese Pharmacopoeia, the ash content of G. lucidum should not be more than $3.2 \%$, while according to the US Pharmacopoeia, ash content should go above $4 \%$. In this study, the ash contents of fruit bodies from 15 samples of Lingzhi did not exceed the specified limit (Table 1).

\section{Water and alcohol soluble extractive}

The result of water and alcohol extractives from fruit bodies are shown in Table 2. According to the 2015 edition of the Chinese and the US Pharmacopoeia, the water-soluble extractive from fruit bodies of G. lucidum should not be less than $3 \%$. Our results showed that the water-soluble extractive of fruit bodies from 15 samples of Lingzhi exceeded the specified limit; the lowest water-soluble extractive was observed in 16ZHJ03-02 (6.5\%), and the highest was in 16SHD01$00(16.43 \%)$.

The Chinese Pharmacopoeia did not stipulate the limit of alcohol-soluble extractive of G. lucidum, while US Pharmacopoeia indicated that the alcohol-soluble extractive of G. lucidum should not be less than $2 \%$. The results showed that the alcohol-soluble extractive of fruit bodies from 15 samples all met the specified limit; the highest alcohol-soluble extractive was $6.39 \%$ in 16 SHD01-00, and the lowest was $2.45 \%$ in 16FJ01-00.

\section{Polysaccharides content}

According to the Chinese Pharmacopoeia, the polysaccharides content of G. lucidum should not be $<0.9 \%$. Table 3 shows that the polysaccharides contents of fruit bodies from 15 samples of Lingzhi determined by the colorimetric method greatly varied from 1.04 to $3.19 \%$; the highest polysaccharides content was seen in 16SHX02-02 (3.19\%) followed by 16SHX01-00 (2.23\%), 16ZHJ01-01 (1.91\%), 16ZHJ03-00 (1.89\%), and 16ZHJ02$00(1.75 \%)$, respectively. The polysaccharides contents determined by HPLC method varied significantly from 0.72 to $2.51 \%$; the lowest polysaccharides content was seen in 16SHX02-00 (0.72\%), and the highest was $2.51 \%$ in 16SHX02-02, followed by 16ZHJ03-00, 16ZHJ01-01, $16 \mathrm{SHX} 01-00$, and 16ZHJ02-00 (1.95\%, 1.87\%, 1.65\%, and $1.53 \%$, respectively).

According to the US Pharmacopoeia, polysaccharides content should not be less than $0.7 \%$ by PMPHPLC method. The monosaccharide composition of these polysaccharides of fruit bodies from 15 samples of Lingzhi was mainly glucose after comparing and analyzing by PMP-HPLC method. The monosaccharide content of polysaccharides extracted from 16FJ01-00, 16SHD01-01, 16SHD01-02, 16SHX01-00, 16SHX0200, 16ZHJ01-00, 16ZHJ01-01, 16ZHJ02-00, 16ZHJ0301 , and $16 \mathrm{ZHJ03-02}$ from high to low were glucose, galactose, mannose, glucuronic acid, and fucose. The monosaccharide content of polysaccharides extracted 


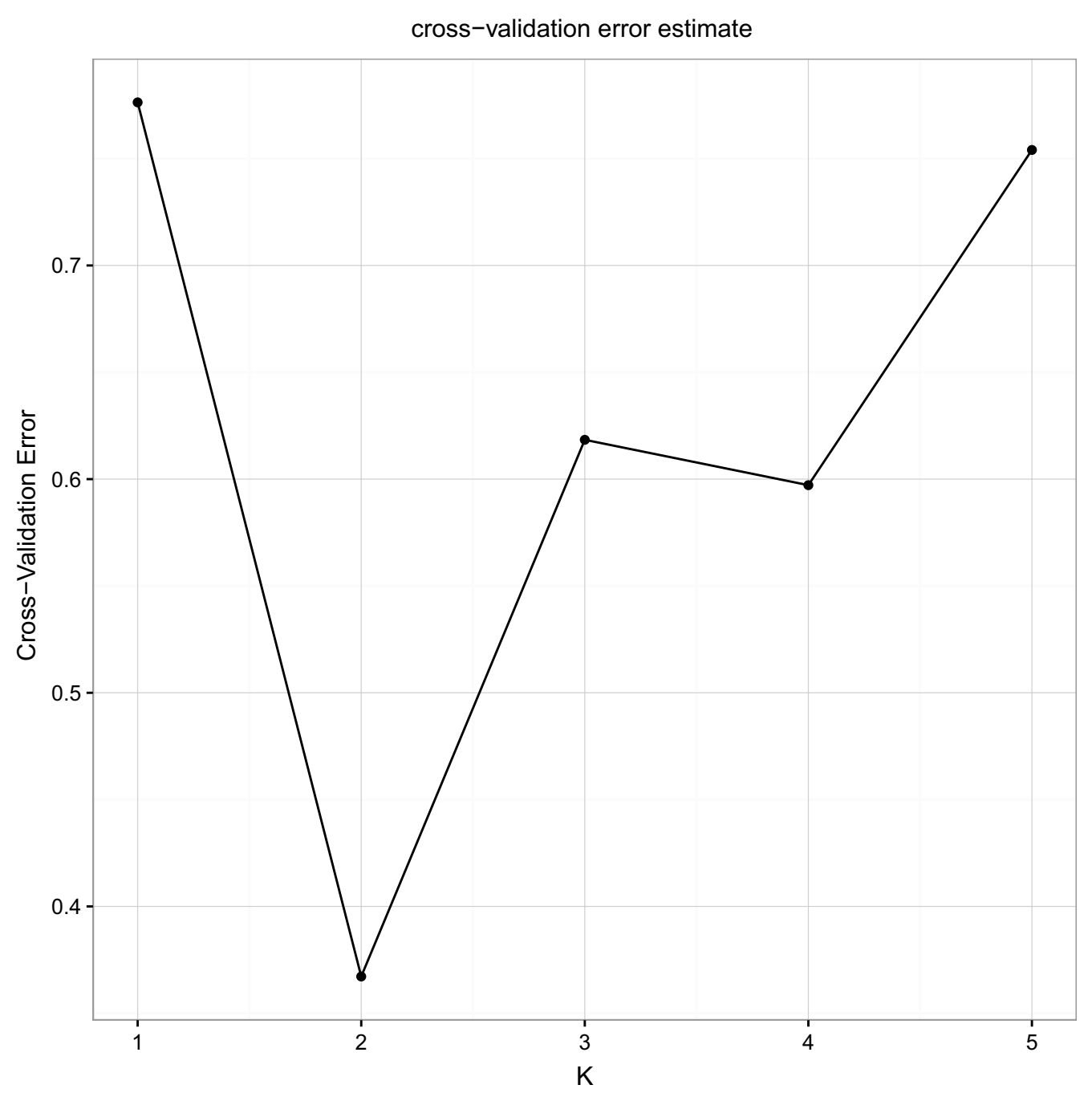

Fig. 4 Analysis of the cross validation error corresponding to different $K$ values

from 16ANH01-00 and 16ANH02-00 from high to low were glucose, mannose, galactose, glucuronic acid, and fucose. The monosaccharide content of polysaccharides extracted from 16SHD01-00, 16SHX02-02, and $16 \mathrm{ZHJ03-00} \mathrm{from} \mathrm{high} \mathrm{to} \mathrm{low} \mathrm{were} \mathrm{glucose,} \mathrm{mannose,}$ galactose, fucose, and glucuronic acid from high to low. As shown in Table 3, the polysaccharides contents of 15 samples of Lingzhi were different in the range of 0.33$0.95 \%$, the lowest polysaccharides content was $0.33 \%$ in 16SHD01-02, and the highest polysaccharides content was $0.95 \%$ in 16 SHD01-00.

As shown in Fig. 6, the polysaccharides content extracted from these samples was quite different, which might be related to regions and growing climatic conditions. The detection method can affect the value, but the trend of the polysaccharide content of each sample was almost the same.

\section{Triterpenoids content}

According to the Chinese Pharmacopoeia, the triterpenoids content of fruit bodies from G. lucidum should not be less than $0.5 \%$. Table 4 shows that the triterpenoids content of fruit bodies from 15 samples of Lingzhi determined by the colorimetric method was different in the range of $0.70-1.74 \%$. The lowest triterpenoids content was seen in 16FJ01-00 (0.70\%), and the highest in 16SHD01-00 (1.74\%), followed by 16ANH01-00, 16ANH02-00, 16SHX02-02 and 16SHD01-01 (1.24\%, $1.10 \%, 0.96 \%$ and $0.94 \%$, respectively).

According to the US Pharmacopoeia, the triterpenoids content should not be less than $0.3 \%$. Triterpenoids content determined by the HPLC method was different in the range of $0.05-0.79 \%$; the lowest content was detected in 16SHD01-00 (0.05\%) and the highest in 16SHD01-02 $(0.79 \%)$. Based on the US Pharmacopoeia reference, only 


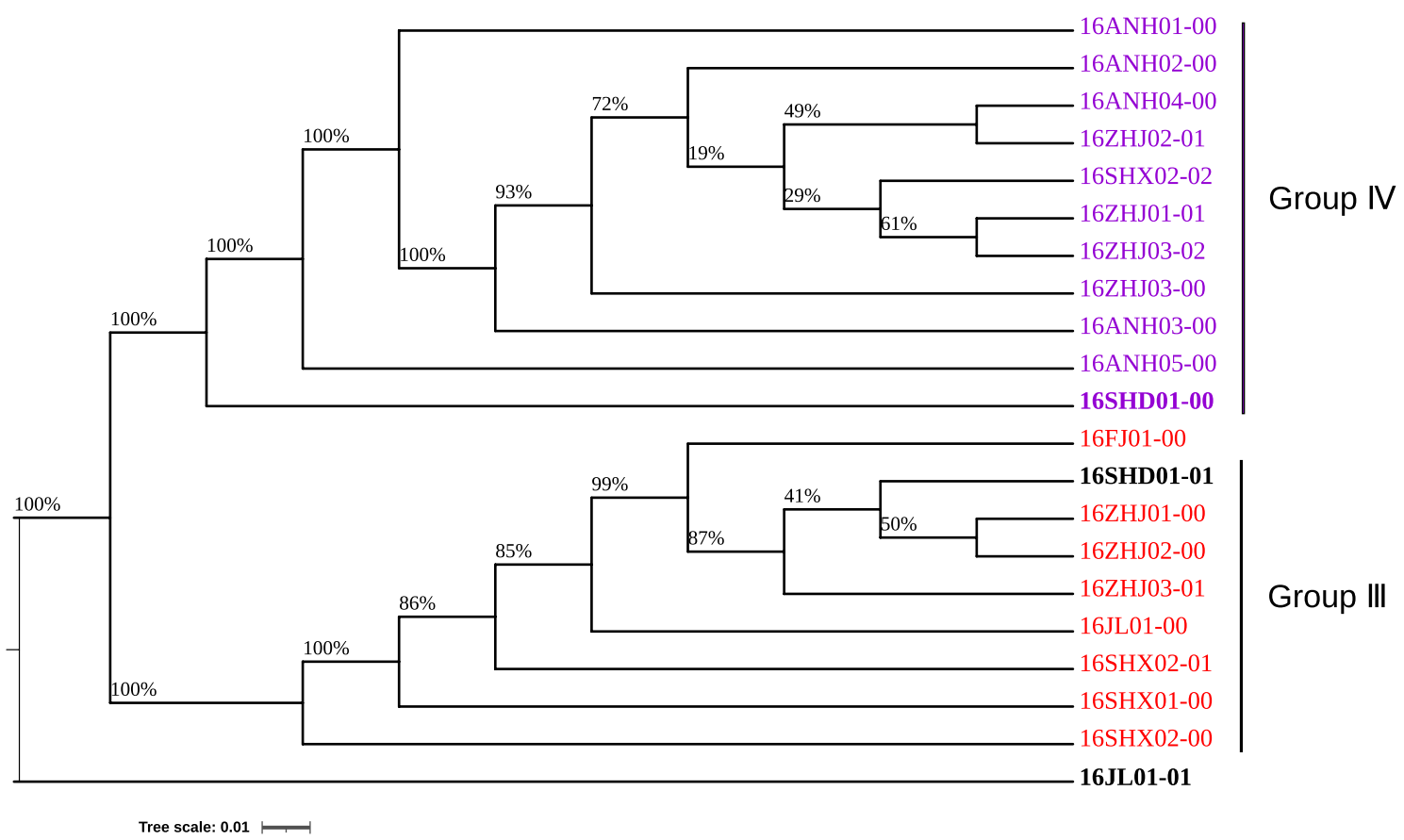

Fig. 5 Phylogenetic tree of 22 lingzhi strains based on SNP

Table 1 The content of moisture and total ash

\begin{tabular}{lll}
\hline Sample & Moisture/\% & Total ash/\% \\
\hline 16ANH01-00 & 4.83 & 1.927 \\
16ANH02-00 & 3.28 & 1.928 \\
16FJ01-00 & 2.32 & 1.928 \\
16SHD01-00 & 3.02 & 1.928 \\
16SHD01-01 & 6.86 & 1.925 \\
16SHD01-02 & 4.03 & 1.927 \\
16SHX01-00 & 1.93 & 1.929 \\
16SHX02-00 & 1.47 & 1.929 \\
16SHX02-02 & 3.93 & 1.927 \\
16ZHJ01-00 & 2.52 & 1.928 \\
16ZHJ01-01 & 1.97 & 1.928 \\
16ZHJ02-00 & 7.12 & 1.925 \\
16ZHJ03-00 & 7.28 & 1.925 \\
16ZHJ03-01 & 2.61 & 1.928 \\
16ZHJ03-02 & 5.88 & 1.926 \\
\hline
\end{tabular}

16SHD01-01, 16SHD01-02, 16SHX01-00, 16SHX02-02, 16ANH01-00, and 16ANH02-00 met the requirement.

As seen in Fig. 7, triterpenoids content extracted from these samples was different. Also, the results of some samples by different detection methods varied greatly. The results obtained by the method of the US Pharmacopoeia were generally low, and most of them did not meet the quality requirements.
Table 2 The results of water soluble and alcohol soluble extract content

\begin{tabular}{lcl}
\hline Sample & $\begin{array}{l}\text { Water-soluble extract } \\
\text { content (\%) }\end{array}$ & $\begin{array}{l}\text { Alcohol-soluble } \\
\text { extract content } \\
\text { (\%) }\end{array}$ \\
\hline 16ANH01-00 & 8.05 & 4.2 \\
16ANH02-00 & 7.93 & 3.41 \\
16FJ01-00 & 8.32 & 2.45 \\
16SHD01-00 & 16.43 & 6.39 \\
16SHD01-01 & 8.56 & 3.4 \\
16SHD01-02 & 10.62 & 3.46 \\
16SHX01-00 & 9.47 & 3.64 \\
16SHX02-00 & 9.31 & 3.19 \\
16SHX02-02 & 13.33 & 3.93 \\
16ZHJ01-00 & 7.38 & 2.74 \\
16ZHJ01-01 & 7.82 & 3.26 \\
16ZHJ02-00 & 9.03 & 2.61 \\
16ZHJ03-00 & 9.27 & 2.88 \\
16ZHJ03-01 & 8.62 & 2.69 \\
16ZHJ03-02 & 6.5 & 2.57 \\
\hline
\end{tabular}

\section{Effect of splenic lymphocyte proliferation}

The proliferation rate of splenocytes treated with polysaccharides extracted from 15 samples of Lingzhi varied from 0.82 to $45.29 \%$ (Fig. 8); the highest proliferation rate was $45.29 \%$ from 16 SHD01-01, followed by 
Table 3 The results of polysaccharides content by colorimetric, HPLC and PMP-HPLC method

\begin{tabular}{llll}
\hline Sample & \multicolumn{2}{l}{ Polysaccharides content (\%) } \\
\cline { 2 - 4 } & $\begin{array}{l}\text { Colorimetric } \\
\text { method }\end{array}$ & HPLC method & $\begin{array}{l}\text { PMP-HPLC } \\
\text { method }\end{array}$ \\
\hline 16ANH01-00 & 1.72 & 1.26 & 0.83 \\
16ANH02-00 & 1.55 & 1.43 & 0.47 \\
16FJ01-00 & 1.07 & 0.83 & 0.51 \\
16SHD01-00 & 1.45 & 1.16 & 0.95 \\
16SHD01-01 & 1.07 & 0.91 & 0.46 \\
16SHD01-02 & 1.18 & 0.98 & 0.33 \\
16SHX01-00 & 2.23 & 1.65 & 0.81 \\
16SHX02-00 & 1.04 & 0.72 & 0.57 \\
16SHX02-02 & 3.19 & 2.51 & 0.9 \\
16ZHJ01-00 & 1.14 & 0.82 & 0.51 \\
16ZHJ01-01 & 1.91 & 1.87 & 0.5 \\
16ZHJ02-00 & 1.75 & 1.53 & 0.55 \\
16ZHJ03-00 & 1.89 & 1.95 & 0.56 \\
16ZHJ03-01 & 1.41 & 0.99 & 0.66 \\
16ZHJ03-02 & 1.41 & 1.35 & 0.39 \\
\hline
\end{tabular}

16ANH01-00, 16ZHJ03-02, 16ZHJ02-00, 16ANH02-00, 16SHD01-02 (38.69\%, 30.06\%, 29.41\%, 26.23\%, 25.17\%, respectively). 16SHX01-00 had the lowest proliferation rate on splenocytes $(0.82 \%)$ (Table 5$)$, the variance of the result was not homogeneous $(\mathrm{P}=0.0124<0.1)$, then Kruskal-Wallis test was conducted, which reveal that most of the samples have significant differences (Additional file 3: Table S8).

\section{Discussion}

G. lingzhi is a large, dark mushroom that promotes health and longevity. Its quality can be affected by many factors such as type of strains, cultivation medium, environment, and artificial management. In this study, we investigated the strains and fruit bodies from the main production areas of G. lingzhi in China using genotyping by sequencing (GBS). The quality of G. lingzhi from each producing area was examined by analyzing genetic diversity, the content of polysaccharides and triterpenoids, and the proliferation rate of splenic lymphocytes treated with polysaccharide extracts from fruit bodies. We found differences in appearance (pileus) of G. lingzhi obtained from different areas. The phylogenetic tree based on ITS sequences revealed the genetic relationship of these Lingzhi strains. 16SHD01-02 was different from other strains, as its sequences match rate was only $16.82 \%$ with the reference genome (Chinese Pharmacopoeia and US Pharmacopoeia). The principal component analysis (PCA) and the phylogenetic tree based on SNP obtained similar results. A cluster of 16SHD01-01 and 16JL01-01 in the phylogenetic tree showed a difference, which may be related to different approaches used to construct the phylogenetic trees. In some studies (Escudero et al. 2014, Wong et al. 2015), the maximum likelihood (ML) method was used to construct phylogenetic trees based on SNPs. However, when we tried this method, the software presented an error prompt and could not complete the phylogenetic tree construction. After testing, it was found that problem was 16SHD01-02, as its genetic relationship was too far from other strains, which was consistent with

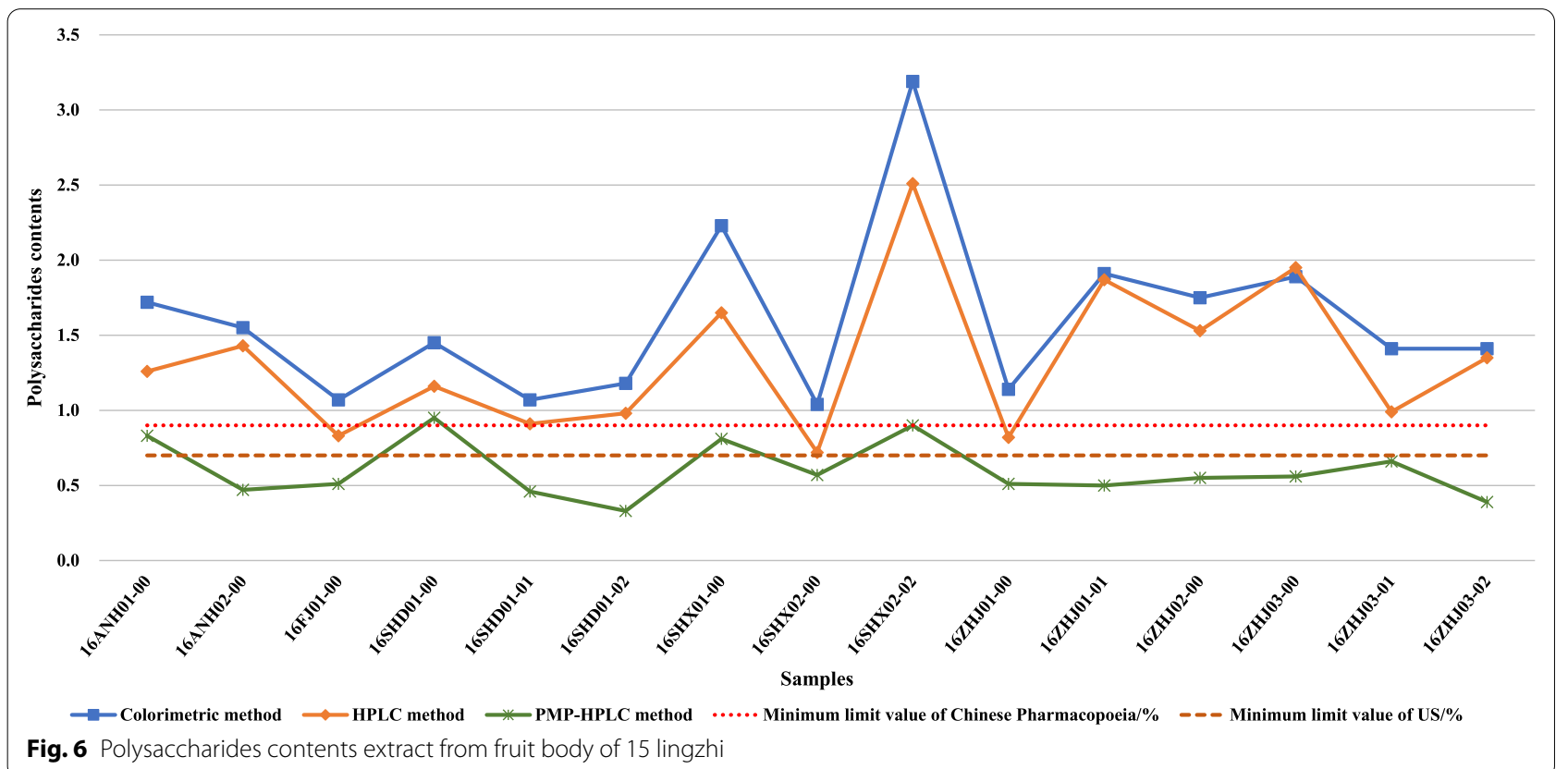


Table 4 The results of triterpenoids content by colorimetric and HPLC method

\begin{tabular}{lll}
\hline Sample & \multicolumn{2}{l}{ Triterpenoids content (\%) } \\
\cline { 2 - 3 } & Colorimetric method & HPLC method \\
\hline 16ANH01-00 & 1.24 & 0.33 \\
16ANH02-00 & 1.1 & 0.44 \\
16FJ01-00 & 0.7 & 0.17 \\
16SHD01-00 & 1.74 & 0.05 \\
16SHD01-01 & 0.94 & 0.63 \\
16SHD01-02 & 0.94 & 0.79 \\
16SHX01-00 & 0.83 & 0.3 \\
16SHX02-00 & 0.78 & 0.17 \\
16SHX02-02 & 0.96 & 0.43 \\
16ZHJ01-00 & 0.73 & 0.22 \\
16ZHJ01-01 & 0.9 & 0.27 \\
16ZHJ02-00 & 0.71 & 0.26 \\
16ZHJ03-00 & 0.86 & 0.29 \\
16ZHJ03-01 & 0.73 & 0.25 \\
16ZHJ03-02 & 0.84 & 0.12 \\
\hline
\end{tabular}

PCA analysis. Finally, it was identified as $G$ sessile by phylogenetic tree based on ITS. This made it clear that there was the wrong strain in the cultivation of Lingzhi.

The components extracted from fruit bodies may be greatly affected by the extraction method. Several polysaccharide extraction methods from G. lingzhi, such as hot water extraction, organic solvent extraction, enzymatic extraction, ultrasonic extraction, and microwave extraction, have been reported (Zhu et al. 2017; Shao et al. 2020). Methods for extracting triterpenoids mainly rely on spectrophotometry and high-performance liquid chromatography (Xu 2014). Some studies suggested that different extraction and detection methods may lead to different results (Yang et al. 2019). Therefore, scientific and accurate methods are necessary to assess the quality of Lingzhi.

In this study, the results of the determination of extract components from the fruit body of Lingzhi revealed that the content of moisture, ash, water-soluble extractive met the specification of Chinese Pharmacopoeia and US. Moreover, the monosaccharide composition of polysaccharides was studied using PMP-HPLC, and the effects of polysaccharides on the proliferation rate of splenocytes were investigated in vitro. The polysaccharides extracted from the fruit body by colorimetric method met the specification of the Chinese Pharmacopoeia. However, only 16SHX02-02, 16SHX01-00, 16ANH01-00, 16SHD01-00 met the polysaccharides content specification of the US Pharmacopoeia as differences in measurement methods. The same phenomenon was observed in the results of triterpenoids content; only 16SHD01-01, 16SHD01-02, 16SHX01-00, 16SHX02-02, 16ANH01-00, and 16ANH02-00 no less than the specification of the US

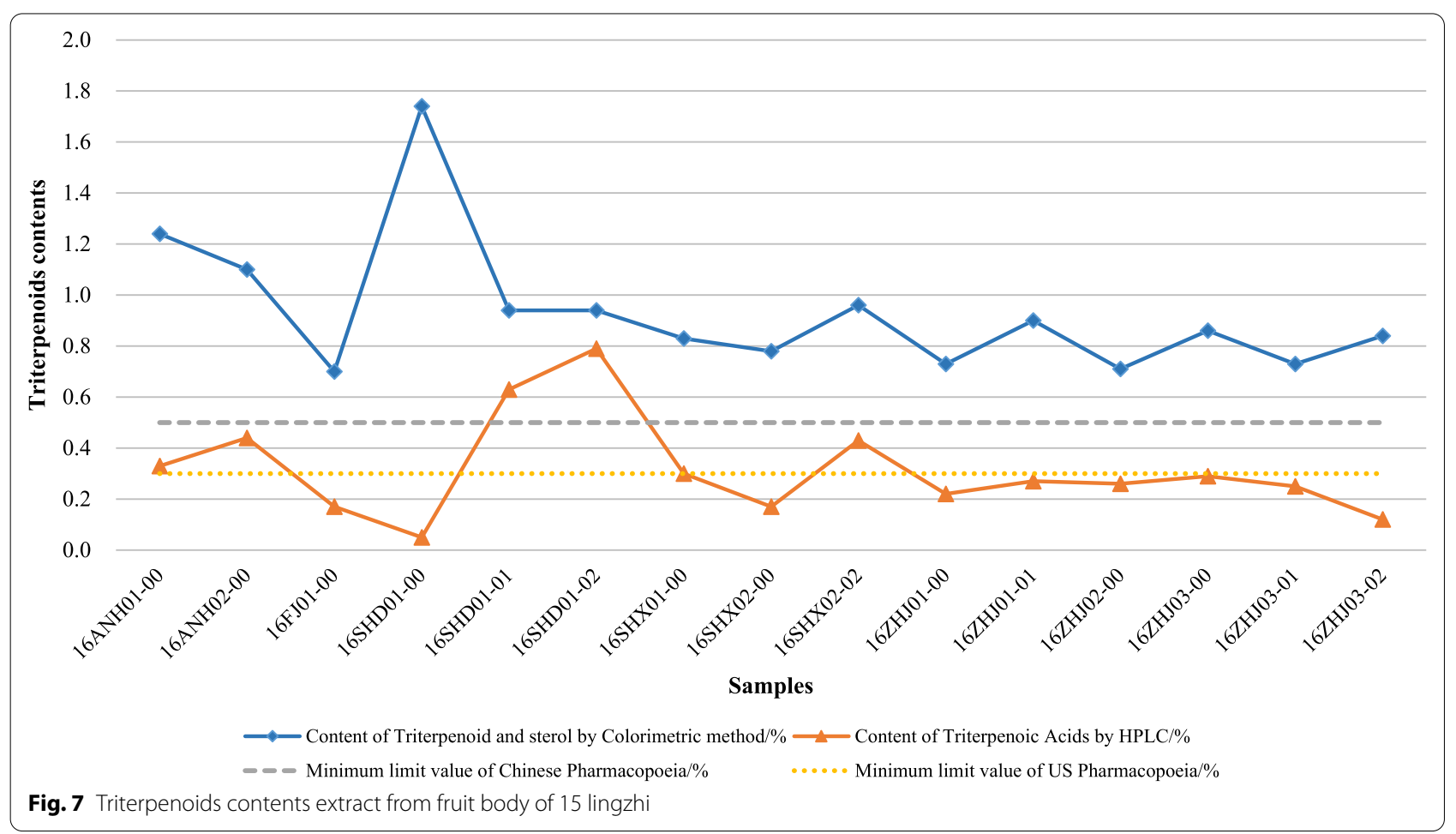




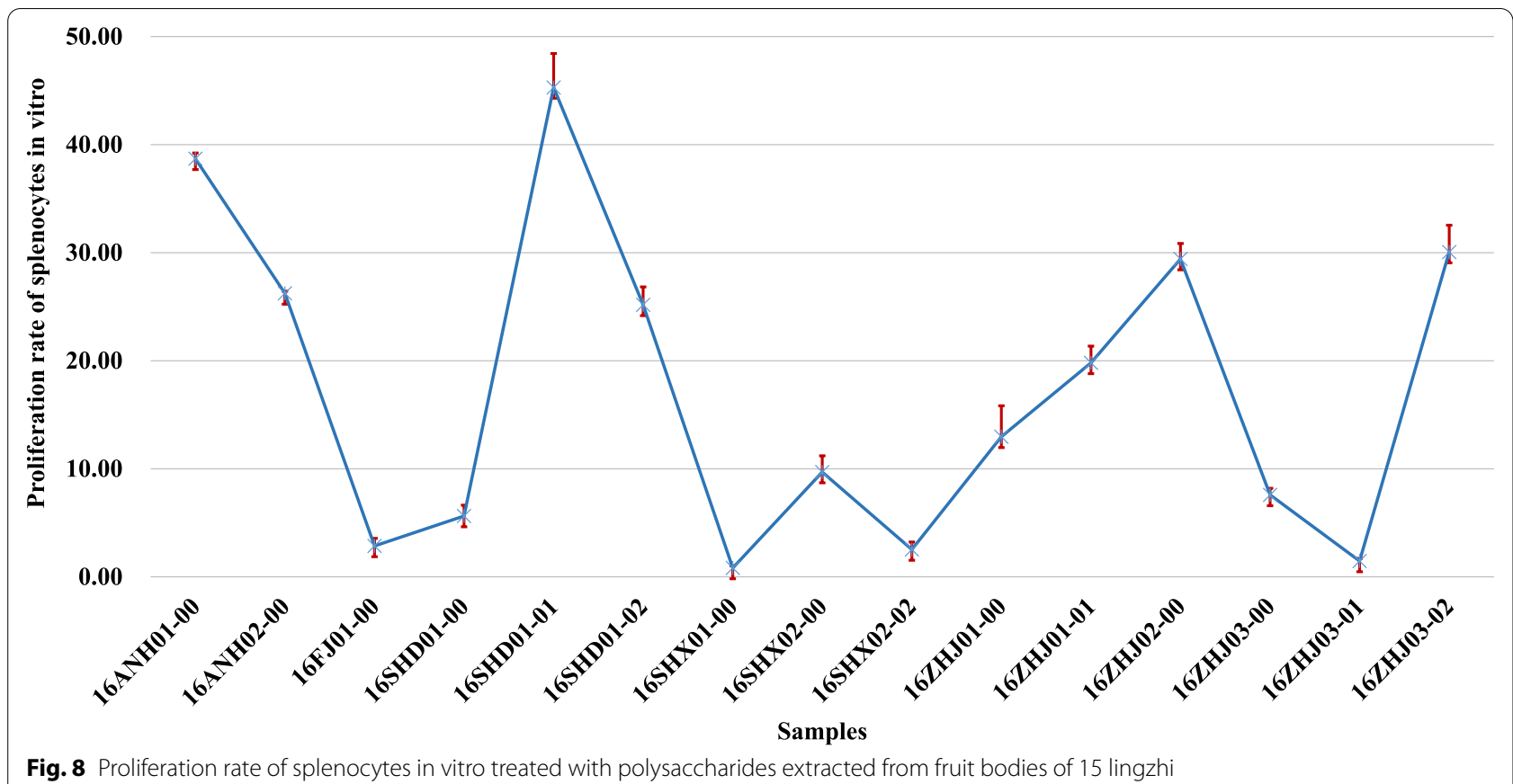

Table 5 Effects of 15 kinds of Lingzhi polysaccharides on the proliferation of splenocytes in vitro

\begin{tabular}{|c|c|c|c|c|c|c|}
\hline \multirow[t]{2}{*}{ Sample } & \multicolumn{5}{|c|}{ Proliferation rate/\% } & \multirow{2}{*}{$\begin{array}{l}\text { Amount of } \\
\text { polysaccharide } \\
\text { ug }\end{array}$} \\
\hline & Value1 & Value2 & Value3 & Mean value & SD & \\
\hline 16ANH01-00 & 38.94 & 39.18 & 37.96 & 38.69 & 0.53 & 10 \\
\hline 16ANH02-00 & 25.99 & 26.48 & 26.23 & 26.23 & 0.2 & 10 \\
\hline 16FJ01-00 & 2.78 & 2.04 & 3.75 & 2.86 & 0.7 & 10 \\
\hline 16SHD01-00 & 4.49 & 6.93 & 5.46 & 5.63 & 1 & 10 \\
\hline 16SHD01-01 & 47.00 & 47.98 & 40.89 & 45.29 & 3.14 & 10 \\
\hline 16SHD01-02 & 27.45 & 23.54 & 24.52 & 25.17 & 1.66 & 10 \\
\hline $16 \mathrm{SHX01-00}$ & 1.07 & 0.82 & 0.58 & 0.82 & 0.2 & 10 \\
\hline $16 \mathrm{SH} \times 02-00$ & 8.64 & 11.82 & 8.64 & 9.7 & 1.5 & 10 \\
\hline $16 \mathrm{SH} \times 02-02$ & 2.04 & 2.04 & 3.51 & 2.53 & 0.69 & 10 \\
\hline 16ZHJ01-00 & 16.95 & 10.35 & 11.57 & 12.96 & 2.87 & 10 \\
\hline 16ZHJ01-01 & 17.68 & 20.37 & 21.35 & 19.8 & 1.55 & 10 \\
\hline $16 Z \mathrm{HJ} 02-00$ & 30.87 & 27.45 & 29.90 & 29.41 & 1.44 & 10 \\
\hline $16 Z$ ZJ03-00 & 6.93 & 7.42 & 8.40 & 7.58 & 0.61 & 10 \\
\hline 16ZHJ03-01 & 1.31 & 1.80 & 1.31 & 1.47 & 0.23 & 10 \\
\hline 16ZHJ03-02 & 33.56 & 28.43 & 28.19 & 30.06 & 2.48 & 10 \\
\hline
\end{tabular}

Pharmacopoeia in triterpenoids content, which revealed that the measurement method had a huge impact on the results. Among the samples, polysaccharides extract from 16SHD01-01, 16SHD01-02, 16ANH01-00, and 16 ANH02-00, which were at the same concentration, showed significant proliferation of splenocytes in vitro.

The content of polysaccharides is usually used as an important indicator of the quality of G. lingzhi. However, the results revealed no correlation between polysaccharide content and its activity; thus, it may be inadequate to focus only on the polysaccharide content and ignore its activity. Therefore, we think that the evaluation of the quality of G. lingzhi requires a comprehensive consideration, as the activity of the active ingredient and its content may be equally important for processors and consumers. 


\section{Abbreviations}

GBS: Genotyping by sequencing; RRL: Reduced-representation libraries; CRoPS: Complexity reduction of polymorphic sequences; RAD-Seq: Restriction-site-associated DNA sequencing; SNP: Single nucleotide polymorphism; GLIS: The active polysaccharides extracted from Lingzhi; RAPD: Random Amplified Polymorphic DNA; ITS: Internal transcribed spacer; ISSR: Inter-simple sequence repeat; SCAR: Sequence characterized amplified regions; SRAP: Sequence-related amplified polymorphism; RFLP: Restriction fragment length polymorphism; AFLP: Amplified fragment length polymorphism; HPLC: High performance liquid chromatography; PMP: 1-Pheny-3-methyl-5-pyrazolone; RPM: Revolution(s) per minute; PCA: Principal component analysis; PBS: Phosphate buffered saline.

\section{Supplementary Information}

The online version contains supplementary material available at https://doi. org/10.1186/s13568-021-01280-y.

\section{Additional file 1: Table S1. Collecting information of Lingzhi} sample(strains and fruit body). Table S2. Table of Genomic electronic digestion assessment statistics. Table S3. Table of base information before and after filter. Table S4. Statistics table of reads filter information. Table S5. HQ clean Reads vs. Reference Genomes. Table S6. Statistical of SNP in samples' chromosome.

Additional file 2. Statistics of annotation results for each sample.

Additional file 3. The homogeneity of variance test and Kruskal-Wallis test of the spleen cell proliferation rate of each sample.

\section{Acknowledgements}

We would like to thank the research and development department of Guangdong Yuewei Edible Technology Co., Ltd. for providing the equipment needed for sample testing, and the Center of Laboratory Animals of the Guangdong Institute of Microbiology for providing the Laboratory and necessary assistance.

\section{Authors' contributions}

$Y L$ performed the molecular biology experiment, was in charge of data processing, and article writing and submission. $\mathrm{XT}, \mathrm{XL}$, and $\mathrm{HH}$ participated in component determination experiments. DC was responsible for genome sequencing. $Y X$ was responsible for sample collection. $X L$ was responsible for cell experiments. CX and LH participated in the processing of partial data. QW was the corresponding author and critically revised the manuscript. All authors read and approved the final manuscript.

\section{Funding}

This study was supported by Key-Area Research and Development Program of Guangdong Province (2018B020205001), Pearl River S\&T Nova Program of Guangzhou (201806010014), and the Science and Technology Planning Project of Shenzhen Municipality (201807010106).

\section{Availability of data and materials}

The data involved in this article can be found in the main manuscript and supplementary data, and the sequencing data have been uploaded to NCBI.

\section{Declarations}

\section{Ethics approval and consent to participate}

The animal study was reviewed and approved by the Institutional Animal Care and Use committee of the Center of Laboratory Animals of the Guangdong Institute of Microbiology. All animal studies were done in compliance with Guangdong Institute of Microbiology institutional animal care regulations and guidelines.

\section{Consent for publication}

Not applicable.

\section{Competing interests}

Author Xiao-cui Tang and Yi-zhen Xie were employed by the company Guangdong Yuewei Edible Fungi Technology Co., Ltd. All authors declare no competing interests.

\section{Author details}

${ }^{1}$ School of Biology and Biological Engineering, South China University of Technology, Guangzhou, China. ${ }^{2}$ Guangdong Provincial Key Laboratory of Microbial Safety and Health, State Key Laboratory of Applied Microbiology Southern China, Institute of Microbiology, Guangdong Academy of Sciences, Guangzhou, China. ${ }^{3}$ Guangdong Yuewei Edible Fungi Technology Co., Ltd., Guangzhou, China.

Received: 17 December 2020 Accepted: 13 August 2021

Published online: 21 August 2021

\section{References}

Chang CJ, Lin CS, Lu CC et al (2017) Corrigendum: ganoderma lucidum reduces obesity in mice by modulating the composition of the gut microbiota. Nat Commun 8:16130. https://doi.org/10.1038/ncomm s16130

Chen S, Xu J, Liu C, Zhu Y, Nelson DR, Zhou S, Li C, Wang L, Guo X, Sun Y, Luo H, Li Y, Song J, Henrissat B, Levasseur A, Qian J, Li J, Luo X, Shi L, He L, Xiang L, Xu X, Niu Y, Li Q, Han MV, Yan H, Zhang J, Chen H, Lv A, Wang Z, Liu M, Schwartz DC, Sun C (2012) Genome sequence of the model medicinal mushroom Ganoderma lucidum. Nat Commun 3:913. https://doi.org/10. 1038/ncomms1923

Chen R, Kang J, Du G, Li B, Liu C, Wang H (2016) Construction of product quality control system of Ganoderma lucidum. Edible Med Mushrooms 24(6):339-344 (in Chinese)

Committee Chinese Pharmacopoeia (2015) Chinese pharmacopoeia-part 1, 2015th edn. China Medical Science and Technology Press, Beijing

Convention The United States Pharmacopeial (2016) The United States pharmacopoe (USP40-NF35), pp 6974-6978

Dai YC, Cao Y, Zhou LW, Wu SH (2013) Notes on the nomenclature of the most widely cultivated Ganoderma species in China. Mycosystema 32(6):947952. https://doi.org/10.13346/j.mycosystema.2013.06.010

Escudero M, Eaton DAR, Hahn M, Hipp AL (2014) Genotyping-by-sequencing as a tool to infer phylogeny and ancestral hybridization: a case study in Carex (Cyperaceae). Mol Phylogenet Evol 79:359-367. https://doi.org/10. 1016/j.ympev.2014.06.026

Hall TA (1999) BioEdit: a user-friendly biological sequence alignment editor and analysis program for Windows 95/98/NT. Nucleic Acids Symp Ser 41:95-98

Jin X, Liu Z, Huang Y, Huang W, Zheng L (2016) Cultivation status and development trend of Ganoderma lucidum in China. Edible Med Mushrooms 24(1):33-37 (in Chinese)

Kaur G, Weinberg R, Milewski AR, Huynh S, Mauer E, Hemmings HC Jr, Pryor KO (2020) Chronic pain diagnosis in refugee torture survivors: a prospective, blinded diagnostic accuracy study. PLOS Med 17(6):e1003108. https://doi. org/10.1371/journal.pmed.1003108

Kumar S, Stecher G, Tamura K (2016) MEGA7: molecular evolutionary genetics analysis version 70 for bigger datasets. Mole Biol Evol 33(7):1870-1874. https://doi.org/10.1093/molbev/msw054

Li H, Durbin R (2009) Fast and accurate short read alignment with BurrowsWheeler transform. Bioinformatics 25(14):1754-1760. https://doi.org/10. 1093/bioinformatics/btp324

Li Y, Zhu ZM, Yao WX, Chen RY (2012) Research status and progress of the triterpenoids in Ganoderma lucidum. Med Plant 3(12):75-81

Li QY, Zhong YY, Chen YX, Zhou WX, Zeng ZJ, Song B (2016) Research advances on the production technology of Ganoderma in China. Edible Fungi China 35(1):8-12. https://doi.org/10.13629/j.cnki.53-1054.2016.01.002 (in Chinese)

Li X, Xie Y, Peng J, Hu H, Wu Q, Yang BB (2019) Ganoderiol F purified from Ganoderma leucocontextum retards cell cycle progression by inhibiting 
CDK4/CDK6. Cell Cycle 18(21):3030-3043. https://doi.org/10.1080/15384 101.2019.1667705

Liao B, Chen X, Han J, Dan Y, Wang L, Jiao W, Song J, Chen S (2015) Identification of commercial Ganoderma (Lingzhi) species by ITS2 sequences. Chin Med 10(1):22. https://doi.org/10.1186/s13020-015-0056-7

Lu J, Qin J, Chen P, Chen X, Zhang Y, Zhao S (2012) Quality difference study of six varieties of Ganoderma lucidum with different origins. Front Pharmacol 3:57. https://doi.org/10.3389/fphar.2012.00057

McKenna A, Hanna M, Banks E, Sivachenko A, Cibulskis K, Kernytsky A, Garimella K, Altshuler D, Gabriel S, Daly M, DePristo MA (2010) The Genome Analysis Toolkit: a MapReduce framework for analyzing next-generation DNA sequencing data. Genome Res 20(9):1297-1303. https://doi.org/10. 1101/gr.107524.110

Paterson RRM (2006) Ganoderma - a therapeutic fungal biofactory. Phytochemistry 67(18):1985-2001. https://doi.org/10.1016/j.phytochem.2006. 07.004

Purcell S, Neale B, Todd-Brown K, Thomas L, Ferreira MA, Bender D, Maller J, Sklar P, de Bakker PI, Daly MJ, Sham PC (2007) PLINK: a tool set for wholegenome association and population-based linkage analyses. Am J Hum Genet 81(3):559-575. https://doi.org/10.1086/519795

Richter C, Wittstein K, Kirk PM, Stadler M (2015) An assessment of the taxonomy and chemotaxonomy of Ganoderma. Fungal Divers 71:1-15. https:// doi.org/10.1007/s13225-014-0313-6

Shao SY, Si XL, Zhang YS, Tu PF, Zhang QY (2020) Recent advances in analytical methods for polysaccharides from edible mushroom. Food Sci 41 (19):272-280. https://doi.org/10.7506/spkx1002-6630-20200512-140 (in Chinese)

Sonah H, Bastien M, Iquira E, Tardivel A, Légaré G, Boyle B, Normandeau É, Laroche J, Larose S, Jean M, Belzile F (2013) An improved genotyping by sequencing (GBS) approach offering increased versatility and efficiency of SNP discovery and genotyping. PLoS ONE 8(1):e54603. https://doi.org/ 10.1371/journal.pone.0054603

Su CL, Tang CH, Zhang JS, Chen MJ, Pan YJ (2007) The phylogenetic relationship of cultivated isolates of Ganoderma in China inferred from nuclear ribosomal DNA ITS sequences. Acta Microbiol Sin 47(1):11-16. https://doi. org/10.13343/j.cnki.wsxb.2007.01.004 (in Chinese)

Sun X, Wang H, Han X, Chen S, Zhu S, Dai J (2014) Fingerprint analysis of polysaccharides from different Ganoderma by HPLC combined with chemometrics methods. Carbohyd Polym 114:432-439. https://doi.org/ 10.1016/..carbpol.2014.08.048

Tang H, Peng J, Wang P, Neil NR (2005a) Estimation of individual admixture: analytical and study design considerations. Genet Epidemiol 28(4):289301. https://doi.org/10.1002/gepi.20064

Tang QJ, Zhang JS, Pan YJ, Reutter W, Fan H (2005b) Activation of normal and tumor-bearing mouse macrophages by active polysaccharide from Ganoderma lucidum. Curr Immunol 25(1):49-52 (in Chinese)

Thompson JD, Gibson TJ, Plewniak F, Jeanmougin F, Higgins DG (1997) The CLUSTAL_X windows interface: flexible strategies for multiple sequence alignment aided by quality analysis tools. Nucleic Acids Res 25(24):48764882. https://doi.org/10.1093/nar/25.24.4876

Vilella AJ, Severin J, Ureta-Vidal A, Heng L, Durbin R, Birney E (2009) EnsemblCompara GeneTrees: complete, duplication-aware phylogenetic trees in vertebrates. Genome Res 19:327-335. https://doi.org/10.1101/gr. 073585.107

Wang K, Li M, Hakonarson H (2010) ANNOVAR: functional annotation of genetic variants from high-throughput sequencing data. Nucleic Acids Res 38(16):e164. https://doi.org/10.1093/nar/gkq603

Wang L, Su HY, Lv SL (2011) Molecular markers and phylogenetic relationships of eight Ganoderma lucidum strains. J Xinxiang Univ 28(03):236-239 (in Chinese)
Wang T, Xie Z, Huang Z, Li H, Wei A, Di J, Xiao H (2015) Total triterpenoids from Ganoderma lucidum suppresses prostate cancer cell growth by inducing growth arrest and apoptosis. Huazhong Univ Sci Technol 35(5):736-741. https://doi.org/10.1007/s11596-015-1499-x

Wasser SP (2014) Medicinal mushroom science: current perspectives, advances, evidences, and challenges. Biomed J 37(6):345-356. https:// doi.org/10.4103/2319-4170.138318

White TJ, Bruns T, Lee S, Taylor J (1990) Amplification and direct sequencing of fungal ribosomal RNA genes for phylogenetics. In: Gelfand DH, Sninsky JJ, Innis MA, White TJ (eds) PCR protocols: a guide to methods and applications. Academic Press, New York

Wong MLM, Gujaria-Verma N, Ramsay L, Yuan H, Caron C, Diapari M, Vandenberg A, Bett KE (2015) Classification and characterization of species within the genus lens using genotyping-by-sequencing (GBS). PLOS ONE 10(3):e0122025. https://doi.org/10.1371/journal.pone.0122025

Wysoker A, Tibbetts K, Fennell T. Picard Tools Version 1.119. 2014. https://sourc eforge.net/projects/picard/files/picard-tools/. Accessed 25 Aug 1999.

Xiao B, Qiu J, Cao P, Gui Y, Lu X, Li Y, Fan L (2014) Development and genetic mapping of SNP markers via genome complexity reduction in tobacco. Acta Agron Sin 40(3):397-404 (in Chinese)

Xu Y. Influence on extraction methods on yields and antioxidant activity of Ganoderma lucidum. Dissertation, Fujian Agriculture and Forestry University. 2014.

Xu MY, Tang CH, Zhang JS, Tang QJ, Yang Y, Jia W, Pan YJ (2008) Development of SCAR markers based on SRAP and ISSR for rapid identification of Ganoderma strains. Mycosystema 27(5):707-717 (in Chinese)

Yan K, Zhang W, Yu H, Wang H (2010) New polysaccharide compounds derived from submerged culture of Ganoderma lucidum and Lycium barbarum. Food Technol Biotechnol 48(1):94-101

Yan Z, Wang H, Liu L, Du G, Chen R (2017) Study on vitro anti-tumor activity of triterpenoids from Ganoderma lucidum. Int J Lab Med 38(5):633-637 (in Chinese)

Yang Z, Han W, Feng N, Zhang J, Wang C, Zhou J, Wang J, Tang Q (2019) Determination of triterpenoids in Ganoderma lingzhi spore powder by HPLC. Mycosystema 38(9):1-9. https://doi.org/10.13346/j.mycosystema. 190196 (in Chinese)

Zhang X. Phenotypic traits analysis and establishment of molecular identities for 17 Ganoderma Lucidum strains. Dissertation, Northeast Agricultural University. 2013. (in Chinese)

Zhao J (1989) New edition of Ganoderma lucidum in China. Science Publishing Company, Beijing (in Chinese)

Zhao H (2020) Study on the quality of four different varieties of Ganoderma Lucidum. J Hubei Minzu Univ 37(2):21-23. https://doi.org/10.13501/j.cnki. 42-1590/r.2020.02.006 (in Chinese)

Zhou LW, Cao Y, Wu SH, Vlasák J, Li D-W, Li MJ, Dai YC (2015) Global diversity of the Ganoderma lucidum complex (Ganodermataceae, Polyporales) inferred from morphology and multilocus phylogeny. Phytochemistry 114:7-15. https://doi.org/10.1016/j.phytochem.2014.09.023

Zhu L, Shi JP, Wang CG, Liu L, Li BG, Zhou J (2017) Research progress of the extract methods and functional properties of Ganoderma lucidum polysaccharides. Mod Chem Ind 37(1):55-59. https://doi.org/10.16606/j.cnki. issn0253-4320.2017.01.013 (in Chinese)

\section{Publisher's Note}

Springer Nature remains neutral with regard to jurisdictional claims in published maps and institutional affiliations. 